Résumés des conférences et travaux

Historiographie médiévale et moderne dans le Saint Empire romain germanique

Nouvelles recherches sur le IX ${ }^{\mathrm{e}}$ siècle et ses sources

\title{
Gerhard Schmitz
}

\section{OpenEdition \\ Journals}

Édition électronique

URL : https://journals.openedition.org/ashp/1004

DOI : 10.4000/ashp.1004

ISSN : $1969-6310$

Éditeur

Publications de l'École Pratique des Hautes Études

Édition imprimée

Date de publication : 2 février 2011

Pagination : 187-191

ISSN : 0766-0677

Référence électronique

Gerhard Schmitz, "Nouvelles recherches sur le IXe siècle et ses sources », Annuaire de l'École pratique des hautes études (EPHE), Section des sciences historiques et philologiques [En ligne], 141 | 2011, mis en ligne le 24 février 2011, consulté le 06 juillet 2021. URL : http://journals.openedition.org/ashp/1004 ; DOI : https://doi.org/10.4000/ashp.1004 


\title{
NOUVELLES RECHERCHES SUR LE IX ${ }^{\text {e }}$ SIÈCLE ET SES SOURCES
}

\author{
Conférences de M. Gerhard Schmitz, \\ vice-président des Monumenta Germaniae Historica, \\ professeur à l'université de Tübingen, \\ directeur d'études invité
}

\section{Le plan de Saint-Gall}

Le plan de Saint-Gall est une source véritablement unique et l'on peine à trouver un document sur lequel il a été autant écrit. Composé de cinq folios de parchemin, de mouton et non de veau, comme on l'a longtemps pensé, pour arriver à un ensemble de 112 sur 77,5 cm, il conserve le plan d'un monastère du haut Moyen Âge. On doit sa conservation non au plan lui-même mais très vraisemblablement au fait qu'un moine du XII ${ }^{\mathrm{e}}$ siècle y a porté au verso la Vita de saint Martin de Tours $(† 397)$.

Une source telle que le plan de Saint-Gall doit être examinée directement; c'est pourquoi son étude scientifique commence véritablement avec la copie que Jean Mabillon en a publiée dans le tome 2 de ses Annales Ordinis S. Benedicti. On dispose désormais, sous l'adresse internet http://www.stgallplan.org/ - une entreprise qui du point de vue technique est pilotée par l'Institute für Advanced Technology in the Humanities de l'University of Virginia, et du point de vue scientifique par l'Universitiy of California (Los Angeles) et l'université de Vienne - d'une excellente reproduction digitale qui montre le plan aussi bien côté recto que côté verso et qui ouvre de nouvelles perspectives d'étude scientifique.

Comme il ressort d'une courte notice de seulement sept lignes, c'est un certain Gozbert qui est le récipiendaire du plan. L'expéditeur ne se nomme pas. Identifier de manière sûre Gozbert est déjà problématique. On pense d'emblée à l'abbé de ce nom, en fonction de 816 à 837 et qui a effectivement fait construire une nouvelle abbatiale dans les années 830, mais cet abbé avait un neveu appelé également Gozbert, actif sur le plan littéraire et en contact avec Walafrid Strabon. Plusieurs érudits le considèrent comme le récipiendaire du plan ou considèrent comme envisageable qu'il en soit ainsi. Les réponses que l'on donne à cette question dépendent en fait de la solution que l'on donne au problème de savoir qui était l'auteur du plan et en quel endroit il a été réalisé. Les réponses fournies sont en fait très divergentes et se concentrent sur un problème de fond : le plan est-il un original ou n'est-il qu'une copie? Cette question est déjà très complexe du simple fait qu'elle implique de définir ce qu'est un « original ». L'on répondra que, dans la mesure où, en tout état de cause, le plan est un unicum, il est aussi, au moins jusqu'à un certain point, un original. C'est d'autant plus le cas que les études de Norbert Stachura ont précisément établi l'existence de ce dont Horn déplorait l'absence : des dessins préliminaires et des piqûres de compas; elles montrent que des esquisses préliminaires ont précédé l'établissement définitif 
du plan. Mais cela ne résout pas le problème : de telles esquisses pourraient s'expliquer par le fait que des modifications ont été apportées à une ébauche intermédiaire sans remettre en cause le fait que le modèle de départ avait un autre format que celui du plan de Saint-Gall dont nous disposons. L'éventail des opinions formulées est très large; il va de l'hypothèse quelque peu exotique d'une origine anglaise (Canterbury) à la thèse du plan comme produit de la réforme monastique sous Louis le Pieux - Benoît d'Aniane serait le véritable inspirateur du plan - en passant par l'idée que la date de naissance du plan remonterait à l'époque de Charlemagne. Dans une toute autre perspective, l'on a également défendu l'idée que les intellectuels de la Reichenau auraient en quelque sorte offert, comme une sorte de jeu intellectuel, un modèle idéal de plan de monastère à l'abbé du monastère voisin dont ils savaient qu'il nourrissait un projet de reconstruction. L'idée d'une origine anglaise n'a quasiment plus de partisans - il faudrait d'emblée supposer la disparition sans aucune trace subsistante de nombreux états intermédiaires du plan; cela vaut également, mutatis mutandis, pour l'idée d'une rédaction originelle du plan à l'époque de Charlemagne. La question du rapport avec le concile réformateur de 816 à Aix-la-Chapelle est plus délicate à trancher : cette réforme monastique était toujours actuelle dans les années 820 et devrait avoir été connue de ceux qui ont conçu le plan. Mais d'un autre côté, on ne peut pas perdre de vue le fait que le plan n'est pas du tout une transcription fidèle des dispositions prises à Aix-la-Chapelle; il manque par exemple le cachot expressément prévu en 816 et à bien d'autres égards encore on ferait violence au plan si on voulait l'interpréter comme parfaitement conforme à la règle de Benoît d'Aniane. La relation avec la Reichenau peut être considérée à présent comme démontrée. Elle était déjà sérieusement envisagée lorsque les études paléographiques de Bernhard Bischoff vinrent lui donner un fondement sûr. Bischoff reconnut deux mains issues de la Reichenau dont celle du bibliothécaire Reginbert. Un autre point d'appui pour cette théorie se trouve dans les fouilles archéologiques d'Alfons Zettler selon lequel les dimensions réelles du monastère de l'île se retrouveraient dans le plan de Saint-Gall. On peut donc considérer comme sûre la thèse d'une origine du plan à la Reichenau. En ce qui concerne la date d'établissement, on peut sans hésiter retenir comme terminus ante quem «avant 830 », date à laquelle Gozbert commença la construction d'une nouvelle abbatiale. Un terminus post quem est moins facile à établir. La thèse la plus fantaisiste vient de Florian Huber qui voulait reconnaître un chronogramme dans l'inscription écrite en majuscules sur le poulailler aux oies et qui aurait pu être déchiffrée en la date de 819 . On a souvent, et à dire vrai avec de bonnes raisons, avancé le nom de l'abbé Haito, ancien évêque de Bâle et abbé de la Reichenau, qui résigna sa charge en 823. Mais cela aide peu à trancher la question de la date d'établissement du plan car Haito peut aussi en tant qu'abbé « honoraire » avoir contribué à la rédaction du plan. À différentes reprises, et récemment encore (Picker), l'on a nommé Walafrid Strabon comme tête pensante derrière la confection du plan; mais une telle proposition se heurte à des problèmes de chronologie : il aurait été encore bien jeune (après son départ à Fulda, il paraît bien n'avoir plus jamais été à la Reichenau) et même si l'on invoque le recours à des formules littéraires, il paraît exclu qu'il ait pu en tant que simple moine s'adresser à l'abbé Gozbert beaucoup plus âgé en le qualifiant de dulcissimus filius. La contradiction est tout de même trop flagrante. En revanche, une telle terminologie convient 
bien à Haito, lequel reste l'auteur le plus probable des lignes de dédicace, et par voie de conséquence de l'ensemble du plan.

Il est quasiment impossible de donner une réponse à la question de savoir si le plan a été conçu dans un esprit d'application à la réalité et s'il était réellement utilisable comme le soutiennent plusieurs (tout particulièrement Hecht). Tout aussi peu assurées me semblent les thèses de Sennhauser selon lesquelles Gozbert se serait effectivement inspiré de modèles architecturaux du plan pour la reconstruction de son abbatiale. Si l'on peut à la rigueur admettre cette thèse, il est en tout cas absolument exclu d'envisager une transposition dans la réalité de l'ensemble du plan. Cela se heurte d'emblée au fait qu'il n'est absolument pas tenu compte de la topographie mais aussi au fait que des infrastructures essentielles telles que l'alimentation en eau ne sont pas du tout prévues. De plus, l'échelle d'ensemble du plan manque de cohérence : les bâtiments annexes ne sont pas représentés à la même échelle que les autres bâtiments. Rien que pour cette raison, le plan ne peut pas être plus qu'une représentation idéale de la vie monastique transcrite sous la forme d'une esquisse. La question de l'échelle est pourtant absolument centrale : à l'intérieur de l'abbatiale figurent en effet en toutes lettres plusieurs indications de mesure. Malencontreusement, les proportions du dessin ne correspondent pas du tout aux mesures indiquées par les inscriptions. Il faudrait selon les échelles retenues agrandir ou rétrécir l'église sans pouvoir faire la même chose avec les autres bâtiments si bien que c'est tout l'ensemble qui perd toute cohérence. L'on n'a pas été en mesure de fournir une solution vraiment satisfaisante pour expliquer ces incohérences apparentes : l'une de ces explications est que l'on aurait transposé les mesures généreuses de l'époque de Charlemagne dans l'époque beaucoup plus modeste et ascétique de Benoît d'Aniane. En tout état de cause, nous ne disposons pas d'un fondement sûr pour calculer la transposition dans la réalité des échelles proposées, étant donné que nous ne connaissons même pas précisément la taille réelle d'un " pied carolingien », en admettant que c'est ce dernier qui devrait être considéré comme l'échelle de référence. Dans ce domaine, la solution la plus convaincante est encore celle de Florian Huber qui part de l'idée d'une continuité jusqu'à l'époque moderne des méthodes antiques d'arpentage et qui peut effectivement expliquer de manière convaincante un certain nombre d'éléments.

En tout état de cause, le plan de Saint-Gall fournit une source étonnante, aussi séduisante que riche, pour des questions touchant à des disciplines très diverses et l'on ne peut être surpris du nombre de « conclusions »-qui se contredisent partiellement entre elles - qui ont été avancées à son propos. Mais du fait de la multiplicité d'approches le plus souvent fragmentaires, il reste nécessaire d'avoir toujours présent à l'esprit une représentation d'ensemble du plan et de s'efforcer de proposer une articulation d'ensemble aux multiples questions restées sans solution (et peut-être insolubles), plutôt que d'ajouter de nouvelles aux anciennes hypothèses portant sur des problèmes partiels.

\section{Nouvelles recherches sur les falsifications pseudo-isidoriennes}

Lorsque Horst Fuhrmann publia en 1972-1974 son œuvre en trois tomes Auftauchen und Verbreitung der pseudoisidorischen Fälschungen, l'on pensait que le 
sujet ne serait pas repris avant longtemps. Mais depuis est intervenue une évolution inattendue du débat érudit si bien qu'il n'est pas inutile de revenir sur l'état de la recherche. Beaucoup de neuf est en effet apparu dans le domaine des textes disponibles.

Cela vaut pour l'Hispana Gallica, l'Hispana Gallica Augustodunensis, les Capitula Angilramni, les Faux capitulaires de Benedictus Levita et également pour les Fausses décrétales. D'une part nous disposons de certaines nouvelles éditions de ces textes, d'autre part des matériaux et des textes ont été fournis qui permettent une approche du domaine beaucoup plus précise. De plus, de nouveaux produits de l'atelier du faussaire pseudo-isidorien ont été édités : les collections du Cod. Phill. 1764, la Collecito Danieliana et les Excerptiones de gestis Chalcedonensis concilii.

C'est à Klaus Zechiel-Eckes que revient une percée décisive sur un problème que l'on croyait jusqu'alors insoluble. Qui était le Pseudo-Isidore ? Il est parvenu à mettre en évidence le fait que plusieurs manuscrits du monastère de Corbie ont été directement utilisés pour l'entreprise de falsification. Mais étant donné que la falsification ne pouvait être réalisée à l'insu, sans l'accord et la direction de l'abbé, le soupçon s'imposait : Paschasie Radbert était le Pseudo-Isidore!

Ainsi se trouve résolue une question absolument décisive mais ce sont du même coup de nouveaux problèmes qui surgissent. En premier lieu, il s'agit de la chronologie interne des faux. Est également revenu sur le devant de la scène le problème du Constitutum Constantini, que les nouvelles recherches de Johannes Fried localisent à Saint-Denis et mettent en relation avec Corbie. Cette thèse également n'a pas fini d'être discutée. En fin de compte, l'on peut dire que tous les problèmes liés à ces faux que l'on a appelés « la plus grande falsification de l'histoire universelle » sont loin d'être réglés.

\section{Les capitulaires : anciennes sources et questions neuves}

Sous le terme de capitulaires, on entend à l'époque carolingienne des édits, décisions, mises en demeure et autres mesures normatives au service de l'administration de l'Empire au sens large du terme. Le nom est une auto-désignation qui est apparue par référence à la forme extérieure de ces documents : un capitulaire se compose de chapitres.

Une division interne en différents registres de thèmes (temporel/ecclésiastique; loi/décret; durable/temporaire, etc.) n'est pas viable : sans ordonnancement rigoureux, la plupart des capitulaires combinent ensemble tous ces registres. Ils trouvent leur origine dans les délibérations des diètes d'empire ou bien dans la volonté du souverain; pour être efficaces, ils doivent être publiés par les missi dans l'Empire. La question de savoir si la proclamation orale ou bien l'expédition écrite fondent la force juridique et contraignante du document est sans objet. Il existe en effet des arguments en faveur des deux positions mais ils ne concernent pas l'époque que l'on peut caractériser comme la transition entre les dispositions normatives (plutôt) orales et les formes d'administration (plutôt) écrites. C'est exactement cela qui se reflète dans l'apparence des capitulaires. 
Parfois, les contemporains n'étaient de toute évidence eux-mêmes pas en mesure de déterminer s'il s'agissait d'un capitulaire ou d'un autre type de document. Ainsi le document n ${ }^{\circ} 47$ de l'édition des Monumenta Germaniae Historica n'est-il pas un capitulaire. Il fut de plus recopié de manière plutôt irréfléchie comme le montre un passage dont la lettre est précisément dépourvue de signification mais dont on peut malgré tout expliquer la formulation.

La tradition manuscrite des capitulaires est tout à fait inégale. Il en est qui sont bien attestés et d'autres que l'on a tout juste conservés en un unique manuscrit. Fait partie de cette dernière catégorie le capitulaire de 802 qualifié par François-Louis Ganshof de « grand capitulaire programmatique » dans lequel Charlemagne aurait donné les fondements de sa nouvelle politique impériale. Il n'est conservé que dans un unique codex du $\mathrm{X}^{\mathrm{e}}$ siècle de provenance haute-italienne et qui se caractérise par son nombre impressionnant de fautes. Le texte est en quelques passages obscur voire absurde. C'est ce qui a conduit à l'idée que cela aurait été la traduction d'une forme subversive de résistance contre les ordres de Charles. Mais on peut réfuter cette opinion en prenant l'exemple de l'interdiction par Charles de forcer d'autres à boire. Certains codices ajoutent ... nisi quantum ei sufficiat. Cela ne fait qu'expliciter ce que Charlemagne voulait obtenir réellement. Il ne voulait pas d'une société d'abstinents; il voulait simplement interdire les beuveries dans lesquelles l'on se soûle. Cet exemple - d'autres pourraient être ajoutés - montre que l'édition des capitulaires réclame la critique textuelle la plus rigoureuse.

\section{Les Francs et l'Empire}

Dans la plupart des études, l'acquisition du titre impérial par Charlemagne est présentée comme un événement extraordinaire et marquant l'avenir. L'on a malgré tout l'impression que les conceptions implicites (parfois aussi explicites) des observateurs des époques ultérieures ne concordent pas vraiment avec celles des contemporains : les Francs avaient aussi peu que Charlemagne lui-même une idée claire de ce que le titre impérial devait véritablement représenter. Le plus souvent, il était lié pour eux à l'Italie; les titres d'imperator ou d'augustus n'étaient au demeurant guère définissables avec précision pour les contemporains. Si l'on met à part la conception d'un cercle relativement étroit d'intellectuels qui s'orientaient essentiellement sur l'idée d'ecclesia - une conception discutée à l'époque de Louis le Pieux et qui n'a pas eu véritablement d'impact dans la réalité - l'Empire est resté une réalité étrangère pour les Francs. Par voie de conséquence, son importance est allé décroissante; il a fini par se réduire à un titre honorifique, traditionnel et passéiste, avant de disparaître et de sombrer avec les petits empereurs italiens du début du $\mathrm{X}^{\mathrm{e}}$ siècle. 